\title{
Attenuation corrected myocardial perfusion SPECT provides powerful risk stratification in patients with coronary artery disease
}

\author{
Ernest V. Garcia, PhD and Fabio P. Esteves, MD
}

\section{See related article, pp. 533-539}

An increasing number of nuclear cardiology laboratories, including ours, image all of their myocardial perfusion SPECT patients using attenuation correction (AC). When compared to non-AC, AC studies generate images with increased linearity between tracer uptake and reconstructed counts yielding increased perfusion defect contrast. ${ }^{1,2}$ This may result in improved detection of left main coronary disease. ${ }^{3} \mathrm{AC}$ also provides higher left ventricular count homogeneity in normal patients ${ }^{4-6}$ because it eliminates the dependence of body habitus on the normal perfusion distribution. AC of diaphragm in men and breast tissue in women results in very similar normal left ventricular counts distribution for males and females. ${ }^{5,6}$ These attributes render AC studies easier to interpret, increasing the confidence of image interpretation $^{7}$ as well as the diagnostic accuracy for obstructive coronary artery disease. ${ }^{3-17}$

Despite the increasing evidence of superior diagnostic accuracy of AC over non-AC in myocardial perfusion SPECT, there is a lack of prognostic data in AC studies. ${ }^{18}$ Non-AC studies have established the role of myocardial perfusion SPECT in noninvasive prognostication of patients with coronary artery disease. ${ }^{19}$ To overcome the lack of literature on AC prognosis, interpreters have had to extrapolate prognostic information from published non-AC studies. This leap of faith becomes worrisome as new imaging protocols with $\mathrm{AC}$ deviate more from the original protocols used in prognostic non-AC studies. This is the case, for instance,

From the Emory University School of Medicine, Emory University Hospital, GA.

Reprint requests: Ernest V. Garcia, PhD, Emory University School of Medicine, Emory University Hospital, 1364 Clifton Rd, NE, Atlanta, GA 30322; ernest.garcia@emory.edu.

J Nucl Cardiol 2009;16:490-2.

$1071-3581 / \$ 34.00$

Copyright $(2009$ by the American Society of Nuclear Cardiology. doi:10.1007/s12350-009-9077-x when one performs non-ECG-gated or stress-only studies. One notable exception to the lack of AC prognostic data is a study by Gibson et $\mathrm{al}^{20}$ using stress-only Tc99m sestamibi AC SPECT in 729 patients with low to intermediate probability for coronary artery disease. The authors showed that in patients with either normal non$\mathrm{AC}$ images or abnormal non-AC but normal AC images the overall cardiac event rate was only $0.6 \%$ during a mean follow-up of $22 \pm 6$ months.

The study by Baghdasarian et $\mathrm{al}^{21}$ in this issue of the journal is another important step in establishing the prognostic value of AC SPECT. The authors examined the prognostic value of non-ECG-gated stress AC SPECT in 419 patients with arrhythmias. They found no difference in the annualized cardiac event rate between $\mathrm{AC}$ and non-AC summed stress scores (SSS) of 0 or greater than 4 but a very significant difference in SSS of $1-3$ (11.4\% for AC vs $2 \%$ for non-AC). In a multivariable analysis, the authors also showed that AC-SSS $>8$ was the most powerful predictor of cardiac events which was incremental over clinical variables.

There are several important points in the study by Baghdasarian et $\mathrm{al}^{21}$ that should be highlighted. First, it shows that AC provides powerful prognostication in a high-risk population. The mean age of the study population was 72 years and $71 \%$ of patients underwent pharmacologic stress testing. Normal non-AC SPECT perfusion studies have been associated with a higher event rate in the elderly population ${ }^{22}$ and pharmacologic stress has been shown to have a doubling of the cardiac event rate as compared to exercise stress. ${ }^{23}$ This is particularly important because it shows that AC prognostication can be trusted in images with relatively higher liver and bowel activity. Second, the pre-defined selection of non-ECG-gated studies only eliminated the potential for biasing the interpreters with the functional analysis. Third, just as in the report by Gibson et al, ${ }^{20}$ the stress-only protocol used provides a cost-effective solution to non-invasive prognostication difficult to duplicate by non-AC stress-only protocols. Fourth and most important, the finding that a SSS of 1-3 was associated with an increased event rate on AC studies and not on non-AC studies underscores both the homogeneity of left ventricular counts distribution with 
$\mathrm{AC}$ in normal patients and the ease of image interpretation. Simply stated, AC-SSS of 0 is normal and everything else is abnormal. Cardiac readers who use AC should be aware that, in contrast to non-AC studies, a SSS of 1-3 is not associated with an event-free shortterm outcome.

As with any high technology imaging modality, careful attention should be paid to quality control in order to reproduce these results in all laboratories. ${ }^{24}$ In the study by Baghdasarian et al, ${ }^{21}$ all attenuation maps were assessed for adequacy of counts, field uniformity, and truncation. Laboratories should be careful to adapt a quality control procedure that is appropriate for the imaging system used and, in particular, to understand the differences in procedures between external transmission source, simultaneous emission/transmission AC and sequential CT/emission AC.

More studies are needed to define the prognostic value of AC SPECT in various patient subgroups. AC is expected to have a more important role in women, in the obese population, and in patients who cannot keep their arms up during image acquisition. AC may also provide added benefit in stress-only and in non-ECG-gated protocols because it is more challenging to differentiate a perfusion defect from attenuation by means of these protocols. AC may have increased value to busy nuclear laboratories where patient throughput is a priority because it obviates the need for prone imaging. Finally, the expertise of the cardiac reader(s) should be also considered to determine the usefulness of AC SPECT. The ease of interpretation of AC images is an attractive option for the inexperienced reader. The nuclear cardiology community should give high priority to determine the prognostic value of ECG-gated rest/stress $\mathrm{AC}$ SPECT studies and how it compares to non-AC studies. For now, the study by Baghdasarian et $\mathrm{al}^{21}$ serves as important evidence that AC myocardial perfusion SPECT is a powerful risk stratification tool in patients with coronary artery disease.

\section{Acknowledgment}

Dr. Garcia receives royalties from the sale of attenuation correction software (ExSPECT II TM $)$ used in the investigation by Baghdasarian et al. ${ }^{21}$ The terms of this arrangement have been reviewed and approved by Emory University in accordance with its conflict of interest policy.

\section{References}

1. Da Silva AJ, Tang HR, Wong KH, et al. Absolute quantification of regional myocardial uptake of $99 \mathrm{mTc}$-sestamibi with SPECT: Experimental validation in a porcine model. $J$ Nucl Med 2001;42:772-9.
2. Garcia EV. SPECT attenuation correction: An essential tool to realize nuclear cardiology's manifest destiny. J Nucl Cardiol 2007; 14:16-24.

3. Duvernoy CS, Ficaro EP, Karabajakian MZ, Rose PA, Corbett JR. Improved detection of left main coronary artery disease with attenuation-corrected SPECT. J Nucl Cardiol 2000;7:639-48.

4. Ficaro EP, Fessler JA, Achermann RJ, et al. Simultaneous transmission-emission thallium-201 cardiac SPECT: Effect of attenuation correction on myocardial tracer distribution. J Nucl Med 1995;36:921-31.

5. Ficaro EP, Fessler JA, Shreve PD, et al. Simultaneous transmission/emission myocardial perfusion tomography. Diagnostic accuracy of attenuation-corrected 99mTc-sestamibi singlephoton emission computed tomography. Circulation 1996;93: 463-73.

6. Grossman GB, Garcia EV, Bateman TM, Heller GV, Johnson LL, Folks RD, et al. Quantitative Tc-99m sestamibi attenuation-corrected SPECT: Development and multicenter trial validation of myocardial perfusion stress gender-independent normal database in an obese population. J Nucl Cardiol 2004;11:263-72.

7. Heller GV, Bateman TM, Johnson LL, et al. Clinical value of attenuation correction in stress-only Tc-99m sestamibi SPECT imaging. J Nucl Cardiol 2004;11:273-81.

8. Gallowitsch HJ, Sykora J, Mikosch P, et al. Attenuation-corrected thallium-201 single-photon emission tomography using a gadolinium-153 moving line source: Clinical value and the impact of attenuation correction on the extent and severity of perfusion abnormalities. Eur J Nucl Med 1998;25:220-8.

9. Hendel RC, Berman DS, Cullom SJ, et al. Multicenter clinical trial to evaluate the efficacy of correction for photon attenuation and scatter in SPECT myocardial perfusion imaging. Circulation 1999;99:2742-9.

10. Links JM, Becker LC, Rigo P, et al. Combined corrections for attenuation, depth-dependent blur, and motion in cardiac SPECT: A multicenter trial. J Nucl Cardiol 2000;7:414-25.

11. Lenzo N, Ficaro EP, Kritzman JN, Corbett JR. Clinical comparison of profile attenuation correction and the Michigan STEP methods. J Nucl Cardiol 2001;8:S19.

12. Shotwell MA, Boyer SK, Chesnut RS, Larkins BA. Improved coronary disease detection with quantitative attenuation-corrected Tl-201 images. J Nucl Cardiol 2002;9:52-62.

13. Slart RH, Tjin HQ, Dirk J, et al. Effect of attenuation correction on the interpretation of $99 \mathrm{mTc}$-sestamibi myocardial perfusion scintigraphy: The impact of 1 year's experience. Eur J Nucl Med Mol Imaging 2003;30:1505-9.

14. Banzo I, Hernandez AR, Pena FJ, et al. Prospective clinical comparison of non-corrected and attenuation- and scatter-corrected myocardial perfusion SPECT in patients with suspicion of coronary artery disease. Nucl Med Commun 2003;24:995-1002.

15. Masood Y, Liu YH, DePuey G, et al. Clinical validation of SPECT attenuation correction using $\mathrm{X}$-ray computed tomography-derived attenuation maps: Multicenter clinical trial with angiographic correlation. J Nucl Cardiol 2005;12:676-86.

16. Utsunomiya D, Tomiguchi S, Shiraishi S, et al. UInitial experience with X-ray CT based attenuation correction in myocardial perfusion SPECT imaging using a combined SPECT/CT system. Ann Nucl Med 2005;19:485-9.

17. Thompson RC, Heller GV, Johnson LJ, Case JA, Cullom J, Garcia EV, et al. Bateman TM: Value of attenuation correction on ECGgated myocardial perfusion imaging related to body mass index. $\mathrm{J}$ Nucl Cardiol 2005;12:195-202.

18. Germano G, Slomka P, Berman DS. Attenuation correction in cardiac SPECT: The boy who cried wolf? J Nucl Cardiol 2007; 14:25-35. 
19. Gibbons RJ. Role of nuclear cardiology for determining management of patients with stable coronary artery disease. J Nucl Cardiol 1994;1:S118-30.

20. Gibson PB, Demus D, Noto R, et al. Low event rate for stress-only perfusion imaging in patients evaluated for chest pain. J Am Coll Cardiol 2002;39:999-1004.

21. Baghdasarian S, Noble G, Ahlberg A, et al. Risk stratification with attenuation corrected Tc-99m sestamibi SPECT myocardial perfusion imaging in the absence of ECG-gating due to arrhythmias. $\mathrm{J}$ Nucl Cardiol (in press, this issue).
22. Steingart RM, Hodnett P, Musso J, et al. Exercise myocardial perfusion imaging in elderly patients. J Nucl Cardiol 2002;9: 573-80.

23. Navare SM, Mather JF, Shaw LJ, et al. Comparison of risk stratification with pharmacologic and exercise stress myocardial perfusion imaging: A meta-analysis. J Nucl Cardiol 2004;11:551-61.

24. Chen J, Caputlu-Wilson SF, Shi H, et al. Automated quality control of emission-transmission misalignment for attenuation correction in myocardial perfusion imaging with SPECT-CT systems. J Nucl Cardiol 2006;13:43-9. 